\title{
Defect-induced negative differential resistance in single-walled carbon nanotubes
}

\author{
G. Buchs, ${ }^{\text {a) }}$ P. Ruffieux, P. Gröning, and O. Gröning \\ EMPA Swiss Federal Laboratories for Materials Testing and Research,nanotech@surfaces, \\ Feuenverkerstrasse 39, CH-3602 Thun, Switzerland
}

(Received 11 July 2008; accepted 2 August 2008; published online 22 August 2008)

\begin{abstract}
The authors report on the observation of a negative differential resistance (NDR) behavior in tunneling spectra recorded on hydrogen and nitrogen plasma-induced atomic defects on semiconducting single-walled carbon nanotubes (SWNTs). The NDR is observed only in the positive bias range of the spectra. This bias asymmetry and the spectral shape in the NDR region can be explained on the basis of a bias-dependent tunneling barrier height model. Within this model the NDR behavior can be directly related to defect-induced sharp electronic states in the SWNT band gap created at the defect sites. (1) 2008 American Institute of Physics. [DOI: 10.1063/1.2975177]
\end{abstract}

The operation of different important electronic components such as Esaki or resonant tunneling diodes relies on negative differential resistance (NDR). ${ }^{1,2}$ With the urge for device miniaturization, NDR has been looked for and found in numerous nanoscale systems (Ref. 3 and references therein), caused by different mechanisms. ${ }^{4-9}$ In this letter, we demonstrate that spatially confined defect sites created on semiconducting single-walled carbon nanotubes (SWNTs) by means of ion bombardment can generate a pronounced NDR behavior in the tunneling spectroscopy. The observed NDR is related to defect-induced narrow and intense electronic gap states and can be explained using a bias-dependent tunneling barrier height model.

The experiments have been carried out in a commercial ultrahigh vacuum scanning tunnel microscope (STM) setup (Omicron LT-STM) operating at $T \simeq 5.5 \mathrm{~K}$, using mechanically cut PtIr tips. Purified HiPco SWNTs (Ref. 10) have been deposited from a sonicated dichloroethane suspension onto atomically flat $\mathrm{Au}(111)$ surfaces. ${ }^{11}$ Defects on the SWNTs have been then created in situ by means of a 2.45 $\mathrm{GHz}$ ECR low energy $\mathrm{H}$ - or $\mathrm{N}$-plasma treatment according to the procedure described in Ref. 11. All presented STM images are treated with the WSXM software. ${ }^{12}$

A STM topography image of a N-plasma treated SWNT can be seen in Fig. 1 with a defect density along the tube axis of about $0.15 \mathrm{~nm}^{-1}$. For both $\mathrm{N}$ and $\mathrm{H}$ ion treatments, the apparent height of the induced defect sites generally ranges from 0.5 to $4 \AA$, with a lateral extension varying between 5 and $30 \AA$. Low temperature scanning tunneling spectroscopy investigations have shown that such defects on semiconducting SWNTs commonly give rise to spatially localized electronic states at different energy positions in the SWNT band gap. ${ }^{13.14}$

Figure 2(b) shows the $I-V$ curves and corresponding $|d I / d V|$ spectra recorded with the lock-in technique at the positions indicated by the red (defect site) and blue (pristine region) triangles on the $\mathrm{H}$-plasma treated SWNT displayed in Fig. 2(a), showing two induced defects (D1, D2). The $|d I / d V|$ spectrum recorded on D2 (red) exhibits typical paired peaks $(\mathrm{P} 1, \mathrm{P} 2)$ related to a $\mathrm{H}$ dimer adsorbed on the SWNT wall. ${ }^{13}$ In Fig. 2(b), a NDR behavior highlighted by

${ }^{3}$ Electronic mail: gilles.buchs@empa.ch. the green shaded zone can be observed in the positive bias range $V>V_{\mathrm{P} 1}$ (bias defined with respect to SWNT potential) of the $I-V$ signal recorded at D2 (red), with the NDR-induced negative value of $d I / d V$ displayed with a reversed positive sign in the recorded $|d I / d V|$ spectrum. Such NDR behavior has been observed only in the positive bias range for the vast majority of the investigated defect-induced gap states with their energetic positions evenly distributed in the positive or negative bias ranges. For gap states at $V<0$ such as $\mathrm{P} 2$, a NDR behavior showed up only in some extremely rare cases, probably due to resonant energy matching between transient discrete levels of the tip and narrow defect-induced peaks in the SWNT (Ref. 15 and references in Ref. 3).

With the typical setpoint parameters used, we confidently assume a shell-tunneling spectroscopy regime in the double barrier tunneling junction formed by the tip, SWNT with defect, and substrate ${ }^{6}$ (Coulomb charging effects are negligible). The NDR behavior can be readily understood within a simple tunneling model. For $T=0 \mathrm{~K}$ and assuming a constant density of states (DOS) for the tip in the bias window of interest, the current resulting from electrons tunneling from the tip into the SWNT defect states can be written as ${ }^{16}$

$$
I(x, y, V) \propto \int_{E_{r}=0}^{e V} \rho_{d}(E, x, y) T(E, e V, z) d E,
$$

where $\rho_{d}(E, x, y)$ is the local density of states (LDOS) of the SWNT at the defect position. Within the Wentzel-KramersBrillouin approximation, the transmission coefficient $T(E, e V, z)$ can be written as ${ }^{16}$

$$
T(E, e V, z)=e^{-\kappa \Sigma \sqrt{\bar{\phi}-E+e V / 2}},
$$

where the decay constant $\kappa$ is given by $\kappa=2\left(2 m_{e} / \hbar^{2}\right)^{1 / 2}$ $=1.02 \AA^{-1} \mathrm{eV}^{-1 / 2} . z$ denotes the tip-substrate distance and $\bar{\phi}=\left(\phi_{d}+\phi_{t}\right) / 2$. with $\phi_{d}$ and $\phi_{t}$ being the SWNT and tip work functions, respectively. For the theoretical investigation of the NDR behavior presented in Fig. 2, we use the hypothetical LDOS of the sample, $\rho_{d}(E, x, y)$, shown in Fig. 3(a). We chose a typical DOS for a semiconducting SWNT with four van Hove singularities (VHSs) defining a $1.2 \mathrm{eV}$ band gap. The defect-induced states are simulated with two Lorentzians (L1, L2) of equal amplitude and an $8 \mathrm{meV}$ full width at half 


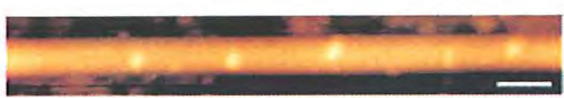

FIG. 1. (Color) STM topography image of a SWNT with N-plasma induced defects; $V_{s}=1.3 \mathrm{~V}, I_{s}=0.12 \mathrm{nA}$, and $T=5.2 \mathrm{~K}$; scale bar: $5 \mathrm{~nm}$.

maximum (FWHM) symmetrically positioned with respect to the midgap level and separated by $0.4 \mathrm{eV}$. The corresponding STM current $I(x, y, V)$ computed from Eq. (1) with $z=7 \AA$ and $\phi_{d}=4.7 \mathrm{eV}$ and $\phi_{t}=5 \mathrm{eV}$ is plotted in Fig. 3(b). The NDR behavior is clearly visible between $V_{\mathrm{L} 1}$ and $V_{\mathrm{VHS} 1}$, whereas the current is continuously increasing in the negative bias range. To understand the nature of the NDR and the positive-negative bias range asymmetry, let us consider the tunneling junction model in Figs. 3(d) and 3(e). For $V>0$ in Fig. 3(d), resonant tunneling occurs when the Fermi level of the tip is aligned with $\mathrm{L} 1$, i.e., for $V=V_{\mathrm{L} 1}$. It results in a current step at $V_{\mathrm{LI}}$ in the $I-V$ curve displayed in Fig. 3(c). The mean tunneling barrier height for electrons tunneling from the tip into $\mathrm{L} 1$ for $V=V_{\mathrm{L} 1}$ is given by $\Phi=\bar{\phi}-e V_{\mathrm{LI}} / 2$. When the bias is further increased, no new states contribute to the tunneling current until $V=V_{\mathrm{VHSI}}=V_{\mathrm{LI}}+\Delta V$ is reached. However, for electrons tunneling into L1, $\Phi$ increases with increasing bias voltage by an amount of $e \Delta V / 2$ at $V=V_{\mathrm{VHSI}}$. This results in a reduced transmission coefficient $T$ and hence, a decreasing current with increasing $V$ in the range $V_{\mathrm{L} 1}<V<V_{\mathrm{VHS}}$, i.e., NDR. Under the assumption FWHM(L1) $\ll \Delta V$, it can be shown from Eqs. (1) and (2) that for $V_{\mathrm{LI}}<V<V_{\mathrm{VHS} 1}$, the ratio $I(V) / I\left(V_{\mathrm{L} 1}\right)$ can be written as

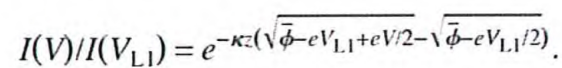

This ratio is always smaller than 1 for $V_{\mathrm{P} 1}<V<V_{\mathrm{VHS} 1}$; thus, NDR is always observed in this bias range due to the bias dependent barrier height. If we assume an ideal discrete state L1, whose spectrum is described by a Dirac peak $\delta(E$ $\left.-e V_{\mathrm{L} 1}\right)$, the current reaches a maximum value $I_{\max }$ at the bias voltage $V=V_{\max }=V_{\mathrm{LI}}$. In reality, the width of the states induces a positive shift in $V_{\max }$. In our example, the value of $V_{\max }$ is shifted by about $42 \mathrm{meV}$ for Ll centered at $E=0.2 \mathrm{eV}$ and $z=7 \AA$. Tunneling through the sufficiently large DOS around narrow states overpowers the current drop and thus impedes the NDR from emerging. This is why no NDR is observed for $V>V_{\mathrm{VHS} 1}$, where a nonzero DOS occurs between the VHSs.

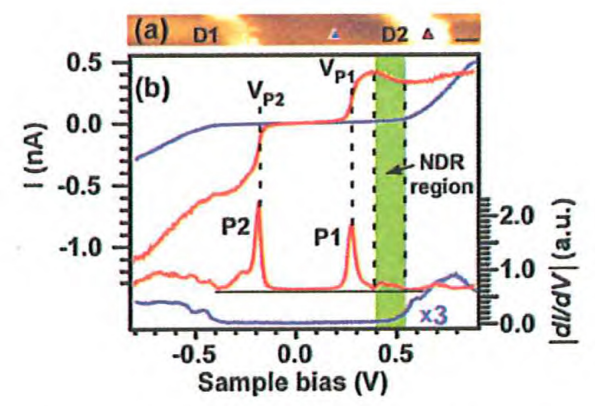

FIG. 2. (Color) (a) STM topography image of a SWNT with two H-plasma induced defects (D1, D2); $V_{s}=1 \mathrm{~V}, I_{s}=0.6 \mathrm{nA}$, and $T=5.6 \mathrm{~K}$; scale bar: 1 $\mathrm{nm}$. (b) $I-V$ curve and $d I / d V$ spectrum recorded at the positions of the red and blue triangles in (a), with a NDR behavior observed on D2 (green shaded region).
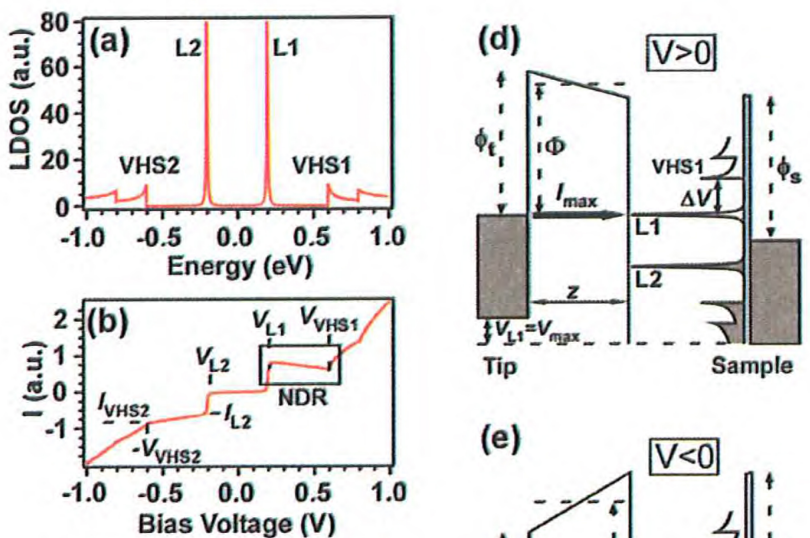

(e)
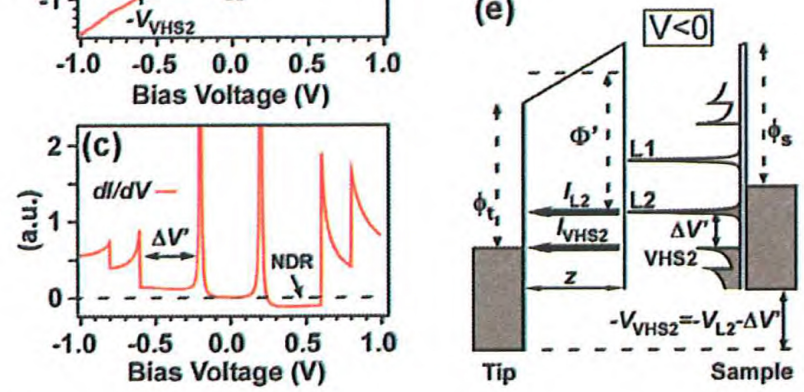

FIG. 3. (Color) (a) Simulated LDOS of a semiconducting SWNT with a double Lorentzian peak structure, L1, L2. (b) Corresponding STM current computed numerically from Eq. (1) with $z=7 \AA$. (c) Derivative $d I / d V$ of the STM current in (b) computed numerically. [(d) and (e)] Schematic of the tip-tube-substrate junction for $V>0$ and $V<0$, respectively. The real proportion in amplitude and width of the Lorentzian peaks L1, L2 is not respected for clarity.

The situation for $V<0$ illustrated in Fig. 3(e) shows that the height of the tunneling barrier $\Phi^{\prime}$ for any isolated state such as L2 continuously decreases with an increase in $|V|$. This results in an increasing tunneling current from L2 into the unoccupied states of the tip for the range $V<V_{\mathrm{L} 2}$ [see Fig. 3(b)]. From the same reasoning above, we can show that for very sharp L2, the ratio $I(V) / I\left(V_{\mathrm{L} 2}\right)$ is always larger than 1 for $V<V_{\mathrm{L} 2}$, thus preventing any NDR behavior from emerging in the negative bias range. Instead, the decrease in $\Phi^{\prime}$ results in a nonzero artifact in the $\Delta V^{\prime}$ region of the $d I / d V$ spectrum displayed in Fig. 3(c), computed numerically from the $I(V)$ curve in Fig. 3(b). This effect has to be

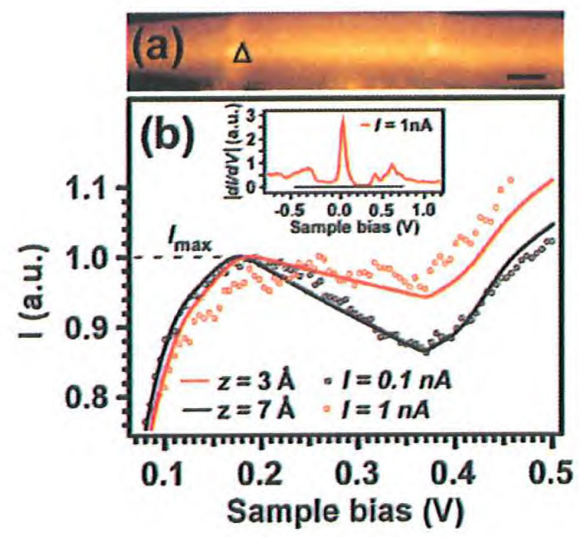

FIG. 4. (Color) (a) STM topography image of a semiconducting SWNT treated with $\mathrm{N}$ plasma: $V_{s}=1 \mathrm{~V}, I_{s}=0.2 \mathrm{nA}$, and $T=5.25 \mathrm{~K}$; scale bar: $2 \mathrm{~nm}$. $\mathrm{f}(\mathrm{b})$ Detail of the NDR region in $I-V$ curves recorded at the position of the black triangle in (a), with fits computed from Eq. (1) with $z=3 \AA$ for $I_{s}=1 \mathrm{nA}$ and $z=7 \AA$ for $I_{s}=0.1 \mathrm{n} \Lambda$. Inset: $d I / d V$ spectrum recorded at the same position for $I_{s}=1 \mathrm{nA}$. 
carefully considered for a consistent analysis of the LDOS around the narrow peaks located in the negative bias range. The apparently higher intensity for the unoccupied states $(V>0)$ with respect to the occupied states $(V<0)$ is mainly due to the weighting of the original LDOS $\rho_{d}(E, x, y)$ by the exponential profile $T$. $^{16}$

From Eq. (2), it follows that the current drop in the NDR region can be tuned by varying the tip-tube distance $z$, resulting in a more pronounced current drop for increasing $z$. This theoretical prediction has been verified experimentally for our system, as shown in Fig. 4. Here we recorded each time five tunneling spectra, once at $0.1 \mathrm{nA}$ and once at $1 \mathrm{nA}$ setpoint current (with $V_{s}=1.2 \mathrm{~V}$ ) at the same position highlighted by the black triangle on a $\mathrm{N}$-plasma-induced defect site shown in the topography image in Fig. 4(a). The averaged and normalized $\left(I_{\max }=1\right) I-V$ experimental data displayed in Fig. 4(b) clearly illustrate the tuning of the NDR by varying the setpoint current. To simulate the NDR behavior, we calculated the current from Eq. (1) with the LDOS being the measured spectrum for $I_{s}=1 \mathrm{nA}$ (inset) divided by the transmission coefficient $T(E=e V, e V, z)$. A measured relative shift of about $5 \mathrm{meV}$ due to band bending ${ }^{3}$ is taken into account in the simulation. The NDR magnitude can be quite well reproduced with $z=7 \AA$ for $I_{s}=0.1 \mathrm{nA}$ and $z=3 \AA$ for $I_{s}=1 \mathrm{nA}$. With a first approximation relation for $z$ derived from Eq. (1) taking into account a contact resistance for $z=0$ of $100 \mathrm{k} \Omega,{ }^{3}$ we find values for $z$ of 4.5 and $5.6 \AA$ for $I_{s}=1$ and $0.1 \mathrm{nA}$, respectively. Even though our approximation of the genuine $\rho_{d}(x, y, E)$ is crude, our simple model fits well with the experimental data.

In conclusion, spatially confined NDR behavior in tunneling $I-V$ curves is observed in the positive bias range on $\mathrm{H}-/ \mathrm{N}$-plasma-induced defect sites on semiconducting SWNTs. Within a bias-dependent tunneling barrier height model, the NDR could be directly related to the very sharp and intense electronic gap states induced by local defects (extend $<1 \mathrm{~nm}$ ). The asymmetry of the NDR behavior (only observed for $V>0$ ) as well as the $z$ dependence of the NDR could be well explained within the same model. It should be stressed that the fact that sharp electronic states can be artificially created in the zero LDOS region of the SWNT band gap represents an ideal situation to generate a NDR by the dependence of the tunneling barrier height. As shown in Fig. 4(b), the NDR can be present in a rather extended voltage range, and although the measurements are carried out at cryogenic temperatures, this effect should persist at room temperature.

The authors would like to acknowledge the financial support by the Swiss National Center of Competence in Research MANEP.

${ }^{\text {I}}$ L. Esaki and P. J. Stiles, Phys, Rev. Lett. 16, 1108 (1966).

${ }^{2}$ L. L. Chang, E. E. Mendez, and C. Tejedor, Resonant Tunneling in Semiconductors: Physics and Applications (Plenum, New York, 1991).

${ }^{3}$ M. Grobis, A. Wachowiak, R. Yamachika, and M. F. Crommie, Appl. Phys. Lett. 86, 204102 (2005).

${ }^{4}$ S. Y. Quek, J. B. Neaton, M. S. Hybertsen, E. Kaxiras, and S. G. Louie. Phys. Rev, Lett. 98, 066807 (2007).

${ }^{5}$ L. Chen, Z. Hu, B. Wang, Y. Luo, J. Yang, and J. G. Hou, Phys. Rev. Lett. 99, 146803 (2007).

${ }^{6}$ P. Liljeroth, L. Jdira, K. Overgaag, B. Grandidier, S. Speller, and D. Vanmaekelbergh, Phys. Chem. Chem. Phys. 8, 3845 (2006).

${ }^{7}$ L. Jdira, P. Liljeroth, E. Stoffels, D. Vanmaekelbergh, and S. Speller, Phys. Rev. B 73, 115305 (2006).

${ }^{8}$ B. J. LeRoy, S. G. Lemay, J. Kong, and C. Dekker, Nature (London) 432, 371 (2004).

${ }^{9}$ A. Zazunov, D. Feinberg, and T. Martin, Phys. Rev. B 73, 115405 (2006)

${ }^{10}$ I. W. Chiang, B. E. Brinson, A. Y. Huang, P. A. Willis, M. J. Bronikowski, J. L. Margrave, R. E. Smalley, and R. H. Hauge, J. Phys. Chem. B 105 , 8297 (2001).

${ }^{11}$ G. Buchs, P. Ruffieux, P. Gröning, and O. Gröning, Appl. Phys. Lett. 90, 013104 (2007).

${ }^{12}$ I. Horcas, R. Fernández, J. M. Gómez-Rodríguez, J. Colchero, J. GómezHerrero, and A. M. Baro, Rev. Sci. Instrum. 78, 013705 (2007).

${ }^{13}$ G. Buchs, A. V. Krasheninnikov, P. Ruffieux, P. Gröning, A. S. Foster, R. M. Nieminen, and O. Gröning, New J. Phys. 9, 275 (2007).

${ }^{14} \mathrm{G}$. Buchs. P. Ruffieux, P. Gröning, and O. Gröning (unpublished).

${ }^{15}$ T.-W. Lyo and P. Avouris, Science 245, 1369 (1989).

${ }^{16} \mathrm{R}$. Wiesendanger, Scanning Probe Microscopy and Spectroscopy: Methods and Applications (Cambridge University Press, Cambridge, 1994). 